\title{
Evolutionary conservation of the circadian gene timeout in Metazoa
}

\author{
Diyan $\mathrm{Li}^{\dagger}$, Yuan $\mathrm{Su}^{\dagger}$, Jianbo Tu${ }^{\dagger}$, Ranlei Wei, Xiaolan Fan, Huadong Yin, Yaodong Hu, \\ Huailiang Xu, Yongfang Yao, Deying Yang and Mingyao Yang
}

Farm Animal Genetic Resources Exploration and Innovation Key Laboratory of Sichuan Province, Sichuan Agricultural University, Chengdu 611130, P. R. China

Submitted: July 21, 2015. Final revision received: October 20, 2015. Accepted: October 29, 2015

\author{
Supplementary Tables
}

$\uparrow$ These authors contributed equally to this work. 
Table S1. CDS length, gene length and intron length of timeout gene in Metazoa

\begin{tabular}{|c|c|c|c|}
\hline Species & Gene Length & Intron Length & CDS Length \\
\hline Mouse & 20878 & 17287 & 3591 \\
\hline Rat & 24041 & 20423 & 3618 \\
\hline Degu & 16890 & 13254 & 3636 \\
\hline Guinea pig & 15653 & 12044 & 3609 \\
\hline Naked mole rat & 24763 & 21133 & 3630 \\
\hline Rabbit & 18117 & 14502 & 3615 \\
\hline Pika & 16624 & 12973 & 3651 \\
\hline Pig & 24981 & 21360 & 3621 \\
\hline Human & 33043 & 29416 & 3627 \\
\hline Gibbon & 14224 & 11662 & 2562 \\
\hline Rhesus & 36723 & 33102 & 3621 \\
\hline Marmoset & 21959 & 18146 & 3813 \\
\hline Tree shrew & 36404 & 32759 & 3645 \\
\hline Yak & 25329 & 21672 & 3657 \\
\hline Cow & 26330 & 22721 & 3609 \\
\hline Sheep & 13239 & 9558 & 3681 \\
\hline Tibetan antelope & 23273 & 19628 & 3645 \\
\hline Dolphin & 13958 & 10334 & 3624 \\
\hline Cat & 30138 & 26514 & 3624 \\
\hline Dog & 28940 & 25286 & 3654 \\
\hline Panda & 13070 & 9449 & 3621 \\
\hline Myotis & 31001 & 27386 & 3615 \\
\hline Horse & 22611 & 18975 & 3636 \\
\hline Hedgehog & 11912 & 8543 & 3369 \\
\hline Manatee & 29675 & 26060 & 3615 \\
\hline Armadillo & 38092 & 34621 & 3471 \\
\hline Opossum & 17116 & 15076 & 2040 \\
\hline Tasmanian devil & 26937 & 23125 & 3812 \\
\hline Platypus & 8503 & 4981 & 3522 \\
\hline Ground-tit & 8627 & 5565 & 3062 \\
\hline Turtle & 2143 & 1585 & 558 \\
\hline Coelacanth & 31128 & 27231 & 3897 \\
\hline Tilapia & 13879 & 10141 & 3738 \\
\hline Platyfish & 13630 & 9703 & 3927 \\
\hline Fugu & 9141 & 5328 & 3813 \\
\hline Zebrafish & 37755 & 33918 & 3837 \\
\hline Spotted gar & 20035 & 16471 & 3564 \\
\hline Sea squirt & 13821 & 10626 & 3195 \\
\hline Domestic silkworm & 33241 & 29467 & 3774 \\
\hline Fruit fly & 75225 & 71070 & 4155 \\
\hline Pea aphid & 11576 & 7205 & 4371 \\
\hline Human body louse & 18334 & 15772 & 2562 \\
\hline Caenorhabditis elegans & 9679 & 5617 & 4062 \\
\hline Guillardia theta & 4489 & 688 & 3801 \\
\hline
\end{tabular}


Table S2. Lineages tested for positive selection by a branch-site model using PAML software

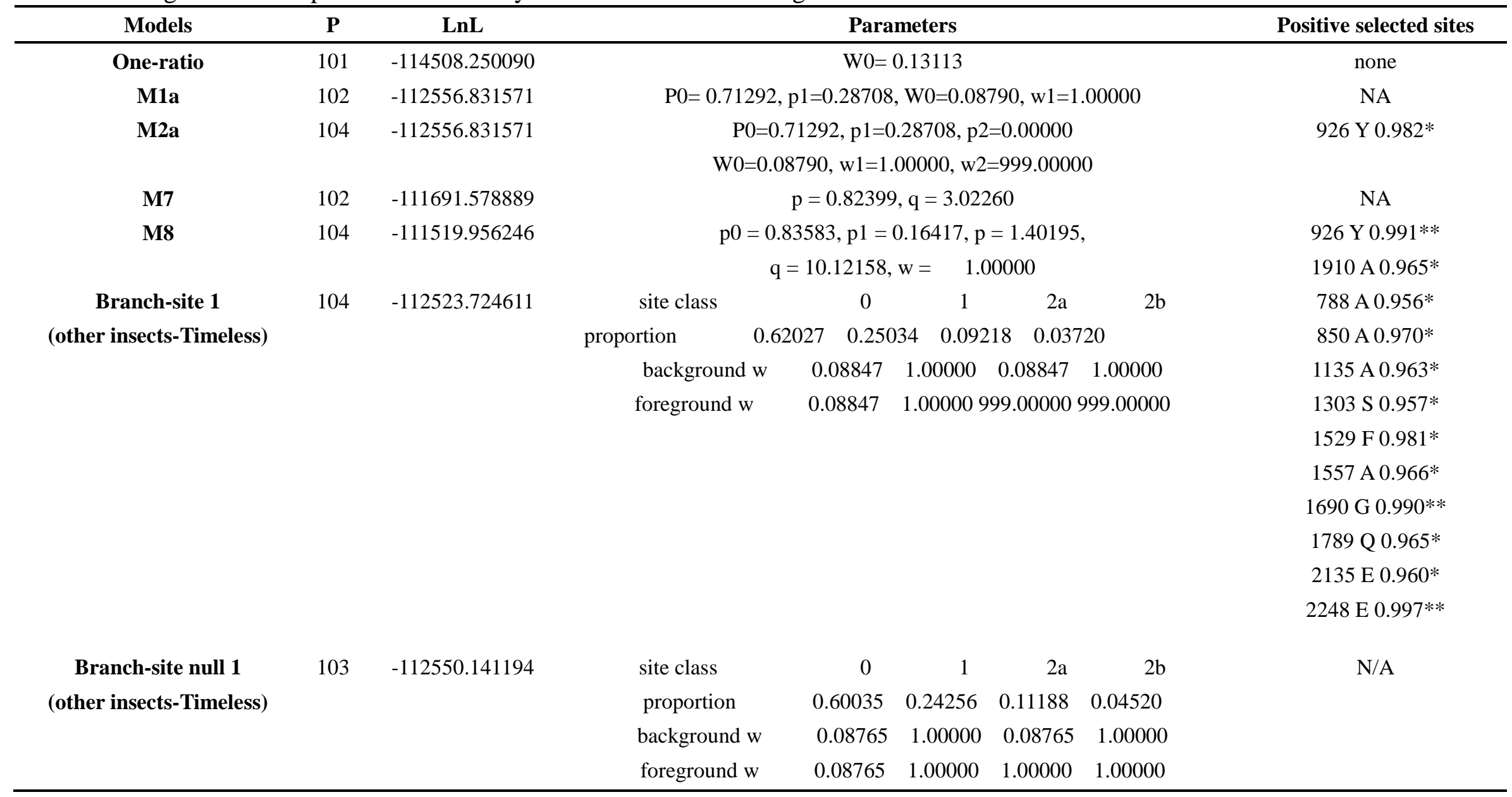




\begin{tabular}{|c|c|c|c|c|c|c|c|}
\hline \multirow{26}{*}{$\begin{array}{c}\text { Branch-site 2 } \\
\text { (Lepidoptera-Timeless) }\end{array}$} & 104 & -112498.658394 & site class & 0 & 1 & $2 a \quad 2 b$ & 132 S $0.955^{*}$ \\
\hline & & & proportion & 0.56439 & 0.22809 & $0.14779 \quad 0.05973$ & 133 A $0.980 *$ \\
\hline & & & background w & 0.08880 & 1.00000 & $0.08880 \quad 1.00000$ & 145 H $0.967 *$ \\
\hline & & & foreground w & 0.08880 & 1.000002 & 20.60439220 .60439 & 158 D $0.983 *$ \\
\hline & & & & & & & 169 Т $0.970 *$ \\
\hline & & & & & & & 197 Q $0.951 *$ \\
\hline & & & & & & & 199 G 0.969* \\
\hline & & & & & & & 255 P $0.975^{*}$ \\
\hline & & & & & & & 297 L 0.954* \\
\hline & & & & & & & 299 Q 0.966* \\
\hline & & & & & & & $341 \mathrm{~K} 0.955^{*}$ \\
\hline & & & & & & & 352 A $0.973 *$ \\
\hline & & & & & & & 373 F $0.970 *$ \\
\hline & & & & & & & 377 S $0.967^{*}$ \\
\hline & & & & & & & $536 \mathrm{R} 0.998^{* *}$ \\
\hline & & & & & & & $539 \mathrm{~V} 0.988^{*}$ \\
\hline & & & & & & & 551 R $0.969^{*}$ \\
\hline & & & & & & & 567 R 0.990* \\
\hline & & & & & & & 635 V 0.982* \\
\hline & & & & & & & 636 R $0.989^{*}$ \\
\hline & & & & & & & 643 C $0.992^{* *}$ \\
\hline & & & & & & & 654 C $0.951 *$ \\
\hline & & & & & & & 662 V 0.971* \\
\hline & & & & & & & 676 L 0.974* \\
\hline & & & & & & & 701 А $0.996^{* *}$ \\
\hline & & & & & & & 738 F $0.991 * *$ \\
\hline
\end{tabular}




\begin{tabular}{c} 
761 T 0.956* \\
762 N 0.987* \\
766 M 0.994** \\
769 T 0.966* \\
771 R 0.961* \\
784 L 0.985* \\
812 T 0.950* \\
845 R 0.998** \\
850 A 0.980* \\
855 F 0.981* \\
894 L 0.973* \\
902 K 0.982* \\
903 R 0.966* \\
931 L 0.999** \\
932 E 0.979* \\
933 A 0.997** \\
950 L 0.969* \\
1130 L 0.978* \\
1135 A 0.990** \\
1287 S 0.991** \\
1291 F 0.998** \\
1334 L 0.976* \\
1340 M 0.998** \\
1342 A 0.984* \\
1348 S 0.955* \\
1355 R 0.997** \\
\hline
\end{tabular}


1359 D 0.972*

1365 A 0.964*

1386 K $0.979 *$

1390 L $0.979^{*}$

1394 N $0.994 * *$

1521 E $0.988^{*}$

1523 Q $0.956^{*}$

1525 L $0.953^{*}$

1536 D $0.981 *$

1564 L $0.999 * *$

1576 E $0.973 *$

1690 G $0.979^{*}$

1698 L $0.968^{*}$

$1782 \mathrm{M} 1.000$ **

1785 E 0.973*

1801 P $0.979 *$

1834 W $0.959^{*}$

1903 L 0.984*

2169 L $0.970 *$

2170 D $1.000^{* * *}$

2193 E $0.978^{*}$

2248 E $0.960^{*}$ 


\begin{tabular}{|c|c|c|c|c|c|c|c|c|}
\hline \multirow{4}{*}{$\begin{array}{c}\text { Branch-site null } 2 \\
\text { (Lepidoptera-Timeless) }\end{array}$} & \multirow[t]{4}{*}{103} & \multirow[t]{4}{*}{-112521.439166} & site class & 0 & 1 & $2 \mathrm{a}$ & $2 b$ & \multirow[t]{4}{*}{ N/A } \\
\hline & & & proportion & 0.53825 & 0.21794 & 0.17354 & 0.07027 & \\
\hline & & & background w & 0.08741 & 1.00000 & 0.08741 & 1.00000 & \\
\hline & & & foreground $\mathrm{w}$ & 0.08741 & 1.00000 & 1.00000 & 1.00000 & \\
\hline Branch-site 3 & 104 & -112532.035506 & site class & 0 & 1 & $2 \mathrm{a}$ & $2 b$ & 453 Q $0.953 *$ \\
\hline (multicellular & & & proportion & 0.53405 & 0.21462 & 0.17928 & 0.07205 & \\
\hline \multirow[t]{2}{*}{ animals-Timeout) } & & & background $\mathrm{w}$ & 0.08784 & 1.00000 & 0.08784 & 1.00000 & \\
\hline & & & foreground $\mathrm{w}$ & 0.08784 & \multicolumn{3}{|c|}{1.00000999 .00000999 .00000} & \\
\hline \multirow{4}{*}{$\begin{array}{l}\text { Branch-site null } 3 \\
\text { (multicellular } \\
\text { animals-Timeout) }\end{array}$} & \multirow[t]{4}{*}{103} & \multirow[t]{4}{*}{-112548.648664} & site class & 0 & 1 & $2 \mathrm{a}$ & $2 b$ & \multirow[t]{4}{*}{ N/A } \\
\hline & & & proportion & 0.55419 & 0.22275 & 0.15911 & 0.06395 & \\
\hline & & & background w & 0.08744 & 1.00000 & 0.08744 & 1.00000 & \\
\hline & & & foreground $\mathrm{w}$ & 0.08744 & 1.00000 & 1.00000 & 1.00000 & \\
\hline \multirow{11}{*}{$\begin{array}{c}\text { Branch-site } 4 \\
\text { (Insecta-Timeout) }\end{array}$} & \multirow[t]{11}{*}{104} & \multirow[t]{11}{*}{-112517.395389} & site class & 0 & 1 & $2 \mathrm{a}$ & $2 b$ & 128 L $0.984 *$ \\
\hline & & & proportion & 0.61670 & 0.24724 & 0.09712 & 0.03894 & 238 Q $0.988^{*}$ \\
\hline & & & background w & 0.08770 & 1.00000 & 0.08770 & 1.00000 & $246 \mathrm{~F} 0.991 * *$ \\
\hline & & & foreground $\mathrm{w}$ & 0.08770 & \multirow{8}{*}{\multicolumn{3}{|c|}{1.00000999 .00000999 .00000}} & 294 Y $0.955 *$ \\
\hline & & & & & & & & 364 L $0.981 *$ \\
\hline & & & & & & & & $368 \mathrm{D} 0.999 * *$ \\
\hline & & & & & & & & $376 \mathrm{~S} 0.995^{* *}$ \\
\hline & & & & & & & & $393 \mathrm{~W} 0.997 * *$ \\
\hline & & & & & & & & 421 Q $0.980^{*}$ \\
\hline & & & & & & & & $471 \mathrm{G} 0.976^{*}$ \\
\hline & & & & & & & & 509 Н $0.963^{*}$ \\
\hline
\end{tabular}




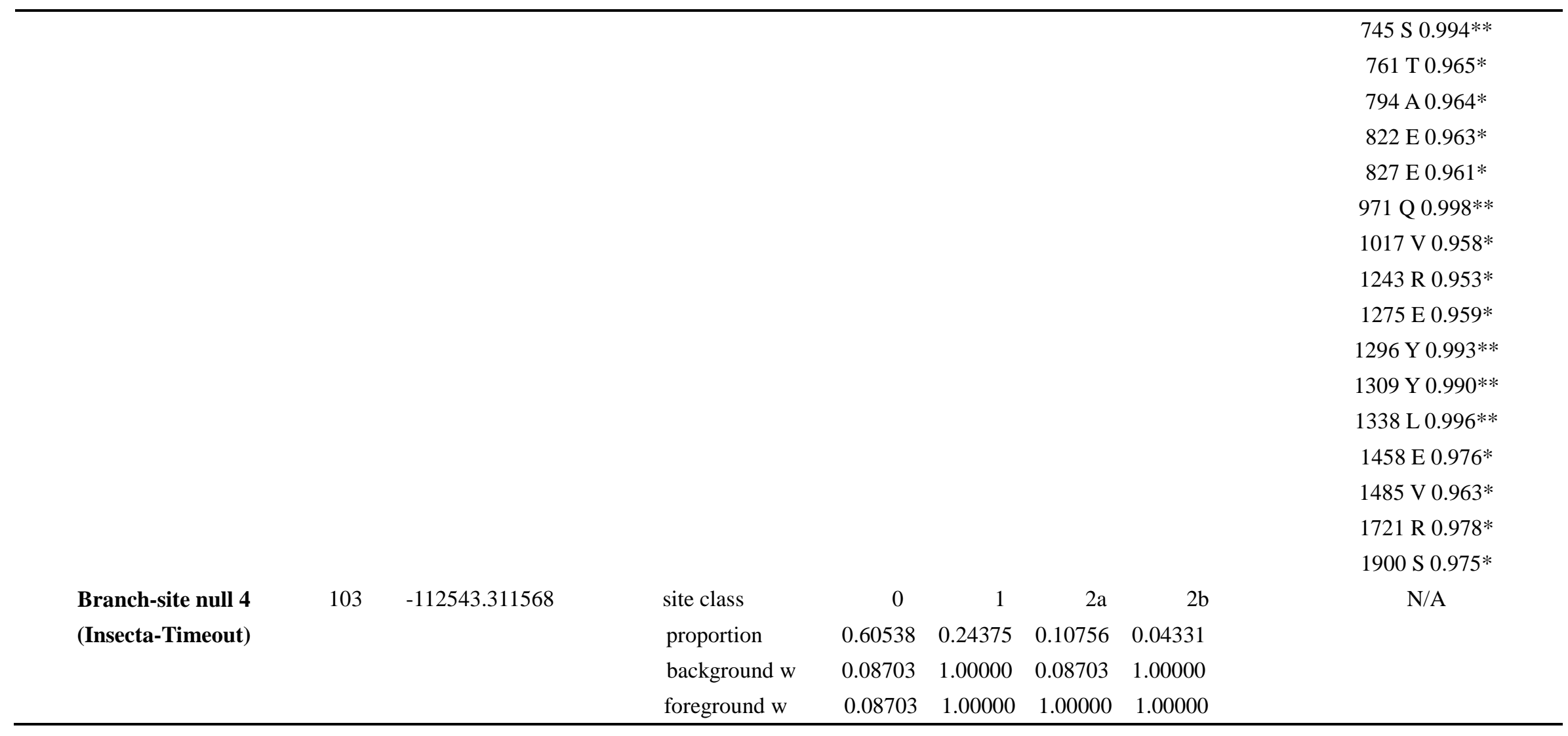




\begin{tabular}{|c|c|c|c|c|c|c|c|c|}
\hline \multirow{6}{*}{$\begin{array}{c}\text { Branch-site } 5 \\
\text { (Vertebrata-Timeout) }\end{array}$} & 104 & -112556.831571 & site class & 0 & 1 & $2 \mathrm{a}$ & $2 b$ & 125 C $0.982 *$ \\
\hline & & & proportion & 0.71292 & 0.28708 & 0.00000 & 0.00000 & 485 G $0.970 *$ \\
\hline & & & background w & 0.08790 & 1.00000 & 0.08790 & 1.00000 & 829 S $0.968^{*}$ \\
\hline & & & foreground w & 0.08790 & 1.00000 & 1.00000 & 1.00000 & 1839 K $0.988^{*}$ \\
\hline & & & & & & & & 1902 S $0.993 * *$ \\
\hline & & & & & & & & 2171 S $0.988^{*}$ \\
\hline \multirow{4}{*}{$\begin{array}{c}\text { Branch-site null } 5 \\
\text { (Vertebrata-Timeout) }\end{array}$} & 103 & -112534.780713 & site class & 0 & 1 & $2 \mathrm{a}$ & $2 b$ & N/A \\
\hline & & & proportion & 0.60864 & 0.24433 & 0.10491 & 0.04212 & \\
\hline & & & background w & 0.08662 & 1.00000 & 0.08662 & 1.00000 & \\
\hline & & & foreground w & 0.08662 & 1.00000 & 1.00000 & 1.00000 & \\
\hline \multirow{5}{*}{$\begin{array}{c}\text { Branch-site } 6 \\
\text { (Mammalia-Timeout) }\end{array}$} & 104 & -112556.831571 & site class & 0 & 1 & $2 \mathrm{a}$ & $2 b$ & 206 S $0.970^{*}$ \\
\hline & & & proportion & 0.71292 & 0.28708 & 0.00000 & 0.00000 & 372 L $0.989 *$ \\
\hline & & & background w & 0.08790 & 1.00000 & 0.08790 & 1.00000 & 735 A $0.978 *$ \\
\hline & & & foreground w & 0.08790 & 1.00000 & 1.00000 & 1.00000 & $901 \mathrm{~K} 0.958^{*}$ \\
\hline & & & & & & & & 1804 S $0.989 *$ \\
\hline \multirow{4}{*}{$\begin{array}{c}\text { Branch-site null } 6 \\
\text { (Mammalia-Timeout) }\end{array}$} & 103 & -112546.285910 & site class & 0 & 1 & $2 \mathrm{a}$ & $2 b$ & N/A \\
\hline & & & proportion & 0.61537 & 0.24744 & 0.09785 & 0.03935 & \\
\hline & & & background w & 0.08668 & 1.00000 & 0.08668 & 1.00000 & \\
\hline & & & foreground w & 0.08668 & 1.00000 & 1.00000 & 1.00000 & \\
\hline
\end{tabular}




\begin{tabular}{|c|c|c|c|c|c|c|c|c|c|}
\hline \multirow{2}{*}{$\begin{array}{c}\text { Branch-site } 7 \\
\text { (Metatheria-Timeout) }\end{array}$} & \multirow[t]{2}{*}{104} & \multirow[t]{2}{*}{-112546.170142} & \multicolumn{2}{|l|}{ site class } & 0 & 1 & $2 \mathrm{a}$ & $2 b$ & $536 \mathrm{R} 0.999 * *$ \\
\hline & & & \multicolumn{2}{|l|}{ proportion } & 0.69977 & 0.27963 & 0.01472 & 0.00588 & \\
\hline & & & \multicolumn{2}{|l|}{ background $\mathrm{w}$} & 0.08709 & 1.00000 & 0.08709 & 1.00000 & \\
\hline & & & \multicolumn{2}{|c|}{ foreground w } & 0.08709 & \multicolumn{3}{|c|}{1.0000011 .3462611 .34626} & \\
\hline \multirow{4}{*}{$\begin{array}{c}\text { Branch-site null } 7 \\
\text { (Metatheria-Timeout) }\end{array}$} & 103 & -112556.831571 & \multirow{2}{*}{$\begin{array}{l}\text { site class } \\
\text { proportion }\end{array}$} & & 0 & 1 & $2 \mathrm{a}$ & $2 b$ & N/A \\
\hline & & & & & 0.71292 & 0.28708 & 0.00000 & 0.00000 & \\
\hline & & & \multicolumn{2}{|l|}{ background w } & 0.08790 & 1.00000 & 0.08790 & 1.00000 & \\
\hline & & & \multicolumn{2}{|l|}{ foreground w } & 0.08790 & 1.00000 & 1.00000 & 1.00000 & \\
\hline \multirow{4}{*}{$\begin{array}{c}\text { Branch-site } 8 \\
\text { (Eutheria-Timeout) }\end{array}$} & 104 & -112548.260565 & site class & & 0 & 1 & $2 \mathrm{a}$ & $2 b$ & $253 \mathrm{~S} 0.975^{*}$ \\
\hline & & & proportion & & 0.70187 & 0.28013 & 0.01287 & 0.00514 & 1785 E $0.968^{*}$ \\
\hline & & & \multicolumn{2}{|l|}{ background w } & 0.08735 & 1.00000 & 0.08735 & 1.00000 & \\
\hline & & & foreground $w$ & 0.08735 & 1.00 & 00126.27 & $496 \quad 126$ & 274 & \\
\hline \multirow{4}{*}{$\begin{array}{c}\text { Branch-site null } 8 \\
\text { (Eutheria-Timeout) }\end{array}$} & 103 & -112553.595412 & \multirow{2}{*}{$\begin{array}{l}\text { site class } \\
\text { proportion }\end{array}$} & & 0 & 1 & $2 \mathrm{a}$ & $2 b$ & N/A \\
\hline & & & & & 0.67531 & 0.27082 & 0.03845 & 0.01542 & \\
\hline & & & background w & & 0.08705 & 1.00000 & 0.08705 & 1.00000 & \\
\hline & & & foreground $\mathrm{w}$ & & 0.08705 & 1.00000 & 1.00000 & 1.00000 & \\
\hline
\end{tabular}

